\title{
Solid-phase Synthesis of Oligodeoxyribonucleoside Boranophosphates by the Boranophosphotriester Method
}

\author{
Mamoru Shimizu, Kazuhiko Saigo, and Takeshi Wada* \\ Department of Medical Genome Sciences, Graduate School of Frontier Sciences, \\ The University of Tokyo \\ Bioscience Building 702, Kashiwa, Chiba 277-8562, Japan
}

Supporting Information 


\section{Table of Contents}

General Information

${ }^{1} \mathrm{H}$ and ${ }^{31} \mathrm{P}$ NMR spectra of compound 6

${ }^{1} \mathrm{H},{ }^{13} \mathrm{C}$ and ${ }^{31} \mathrm{P}$ NMR and IR spectra of compound 7

${ }^{1} \mathrm{H},{ }^{13} \mathrm{C}$ and ${ }^{31} \mathrm{P}$ NMR and IR spectra of compound 10a

${ }^{1} \mathrm{H},{ }^{13} \mathrm{C}$ and ${ }^{31} \mathrm{P}$ NMR and IR spectra of compound $\mathbf{1 0 b}$

${ }^{1} \mathrm{H},{ }^{13} \mathrm{C}$ and ${ }^{31} \mathrm{P}$ NMR and IR spectra of compound 10c

${ }^{1} \mathrm{H},{ }^{13} \mathrm{C}$ and ${ }^{31} \mathrm{P}$ NMR and IR spectra of compound 10d

UV melting curves of $\mathrm{T}_{12} / \mathrm{dA}_{12}, \mathrm{~T}\left({ }_{P B} \mathrm{~T}\right)_{11} / \mathrm{dA}_{12}$,

$\mathrm{d}(\mathrm{CAGT})_{3} / \mathrm{d}(\mathrm{ACTG})_{3}, \mathrm{~d}\left(\mathrm{C}_{P B} \mathrm{~A}_{P B} \mathrm{G}_{P B} \mathrm{~T}\right)_{3} / \mathrm{d}(\mathrm{ACTG})_{3}$,

$\mathrm{d}(\mathrm{CAGT})_{3} / \mathrm{r}(\mathrm{ACUG})_{3}$, and $\mathrm{d}\left(\mathrm{C}_{P B} \mathrm{~A}_{P B} \mathrm{G}_{P B} \mathrm{~T}\right)_{3} / \mathrm{r}(\mathrm{ACUG})_{3}$ 


\section{General Information.}

${ }^{1} \mathrm{H}$ NMR spectra were obtained at $300 \mathrm{MHz}$ with tetramethylsilane (TMS) as an internal standard in $\mathrm{CDCl}_{3}$, with TMS as an internal standard in $\mathrm{CD}_{3} \mathrm{CN}$, and with 2,2,3,3-d $d_{4}-3$-(trimethylsilyl)propionic acid sodium salt $\left(\mathrm{TSP}-d_{4}\right)$ as an external standard in $\mathrm{D}_{2} \mathrm{O} .{ }^{13} \mathrm{C}$ NMR spectra were obtained at $75.45 \mathrm{MHz}$ with $\mathrm{CDCl}_{3}$ as an internal standard $(\delta 77.0)$ in $\mathrm{CDCl}_{3}$, with TMS as an external standard in $\mathrm{CD}_{3} \mathrm{CN}$, and with DSS as an external standard in $\mathrm{D}_{2} \mathrm{O} .{ }^{31} \mathrm{P}$ NMR spectra were obtained at $121.5 \mathrm{MHz}$ with $85 \% \mathrm{H}_{3} \mathrm{PO}_{4}$ as an external standard. Thin layer chromatography (TLC) was performed on TLC plates Silica gel $60 \mathrm{~F}_{254}$. Silica gel column chromatography was carried out using Silica gel 60N (63-210 $\mu \mathrm{m})$. Reversed phase HPLC was

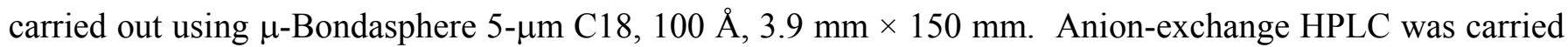
out using Gen-Pak FAX column $(3-\mu \mathrm{m}, 4.6 \mathrm{~mm} \times 100 \mathrm{~mm})$. Organic solvents were purified and dried by the appropriate procedure. Manual solid-phase synthesis was performed by using a glass filter $(10 \mathrm{~mm} \times 50$ $\mathrm{mm}$ ) with a stopper at the top and a stopcock at the bottom as a reaction vessel. 
Supporting Info Page 4
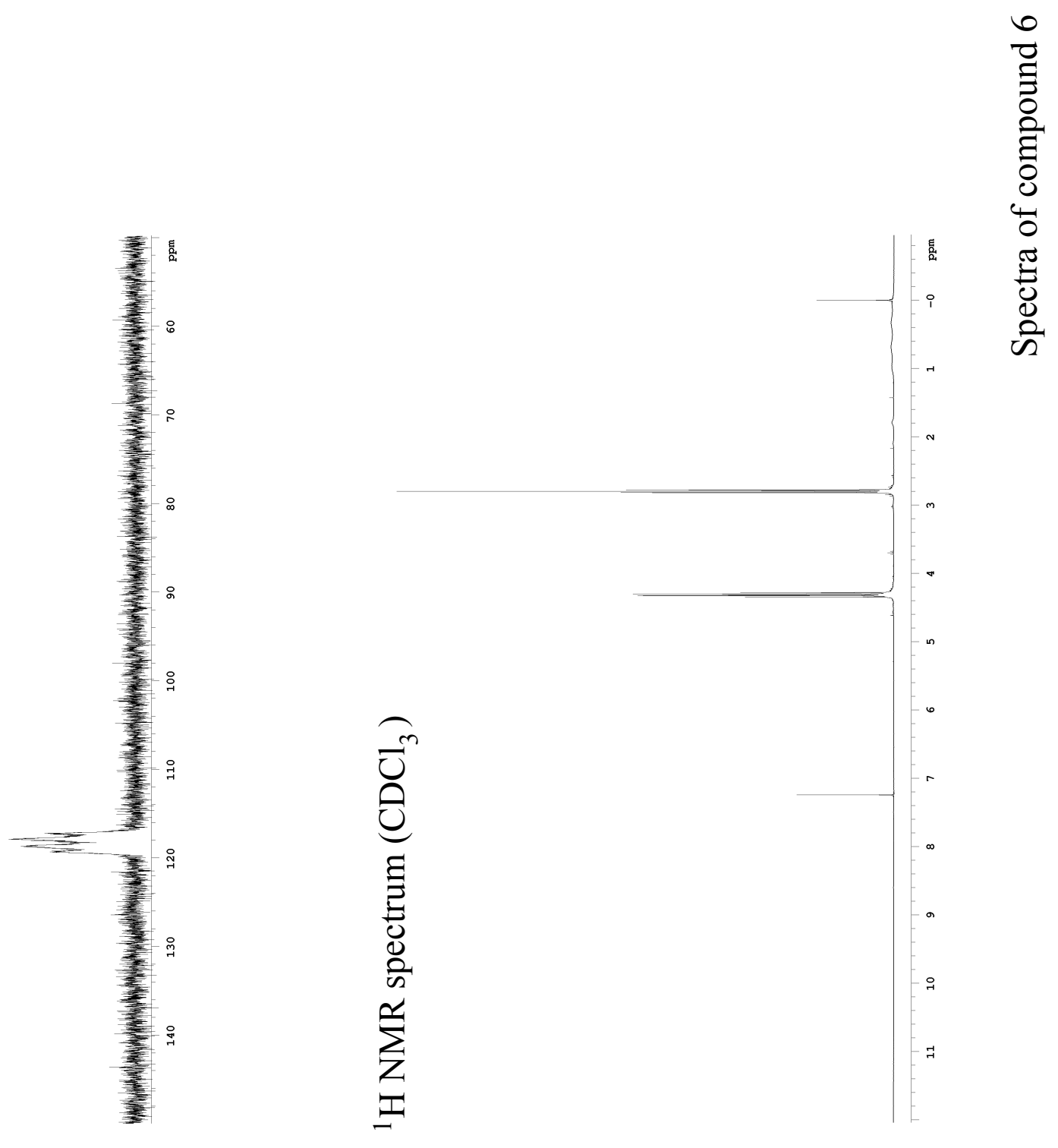


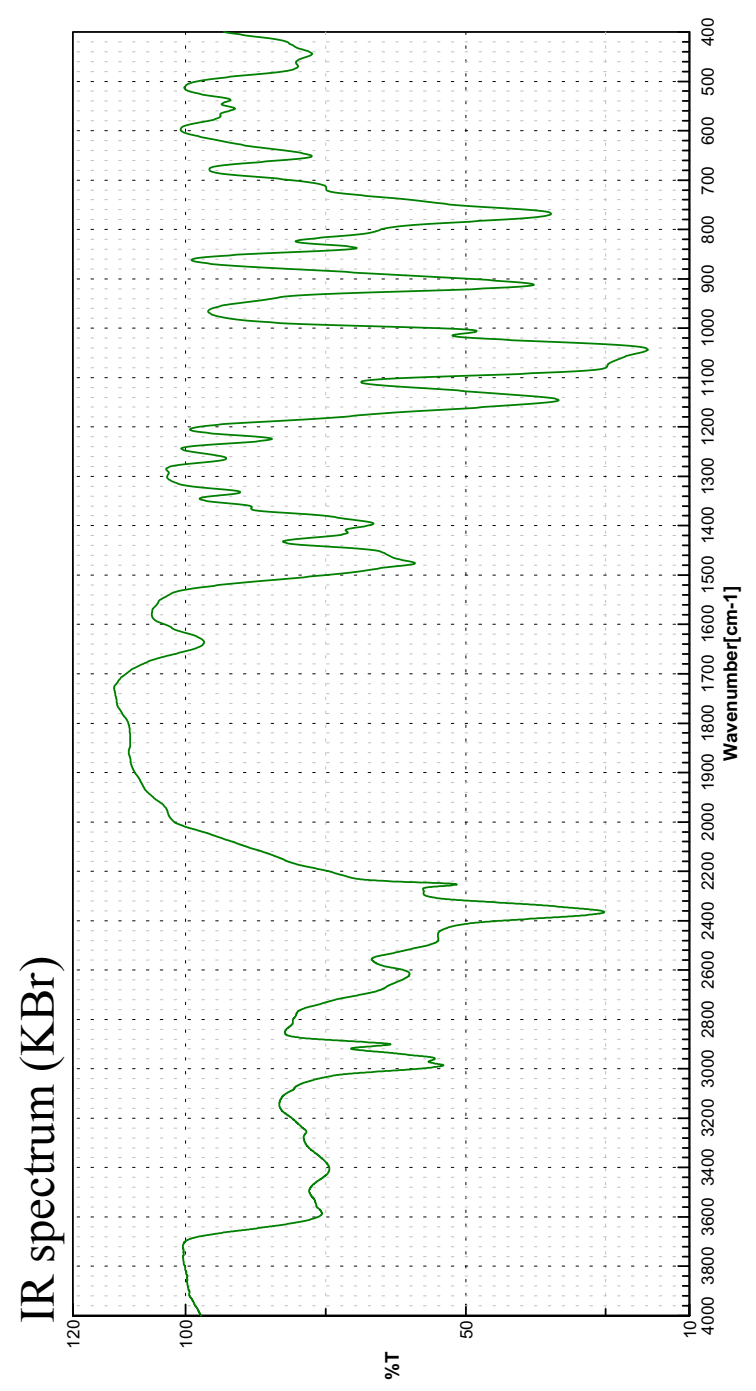

Supporting Info Page 5

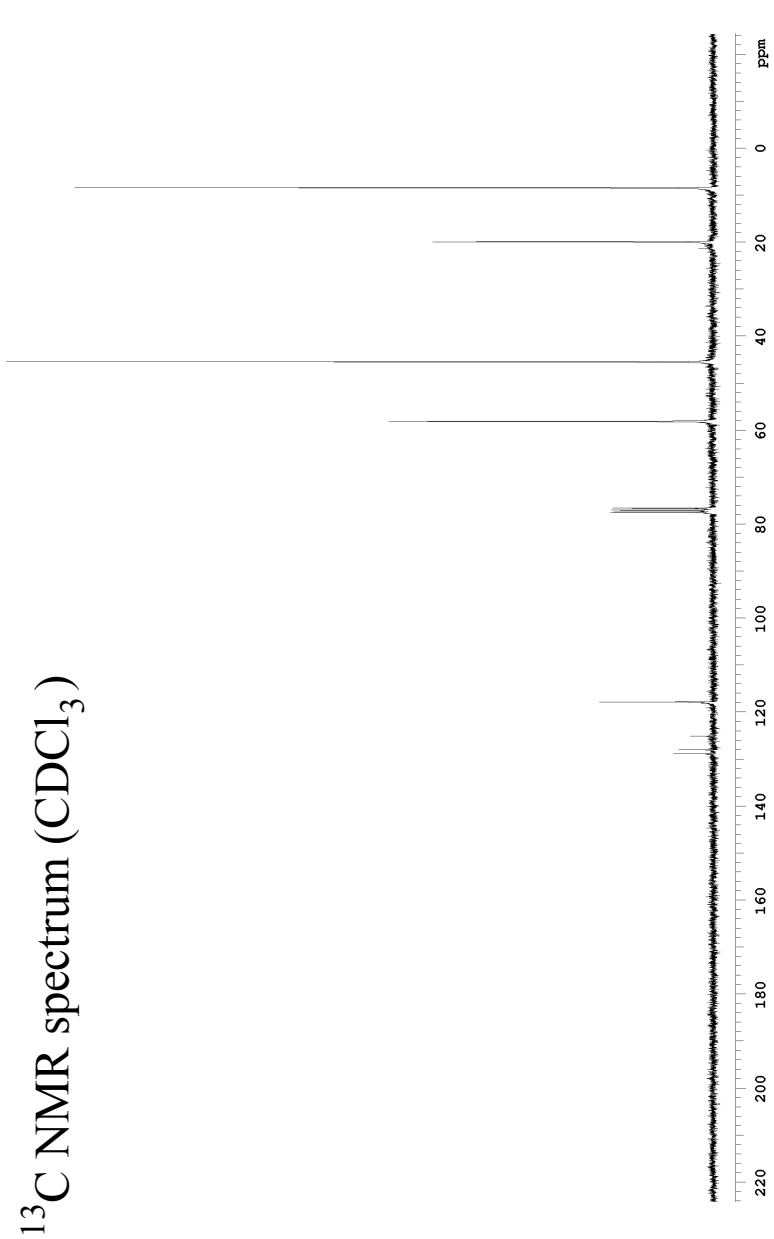

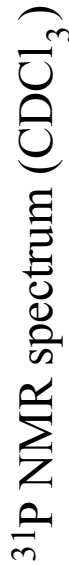

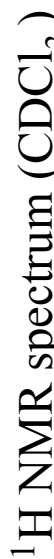




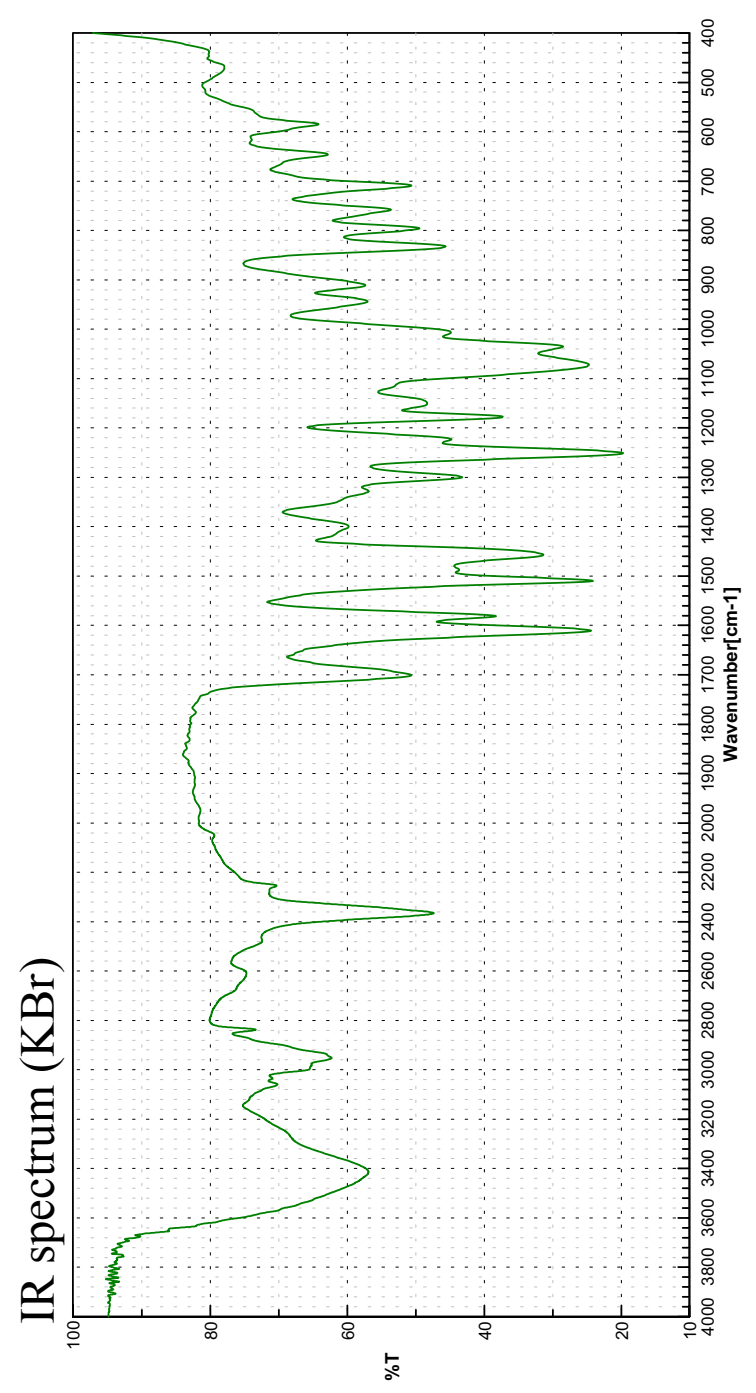

Supporting Info Page 6

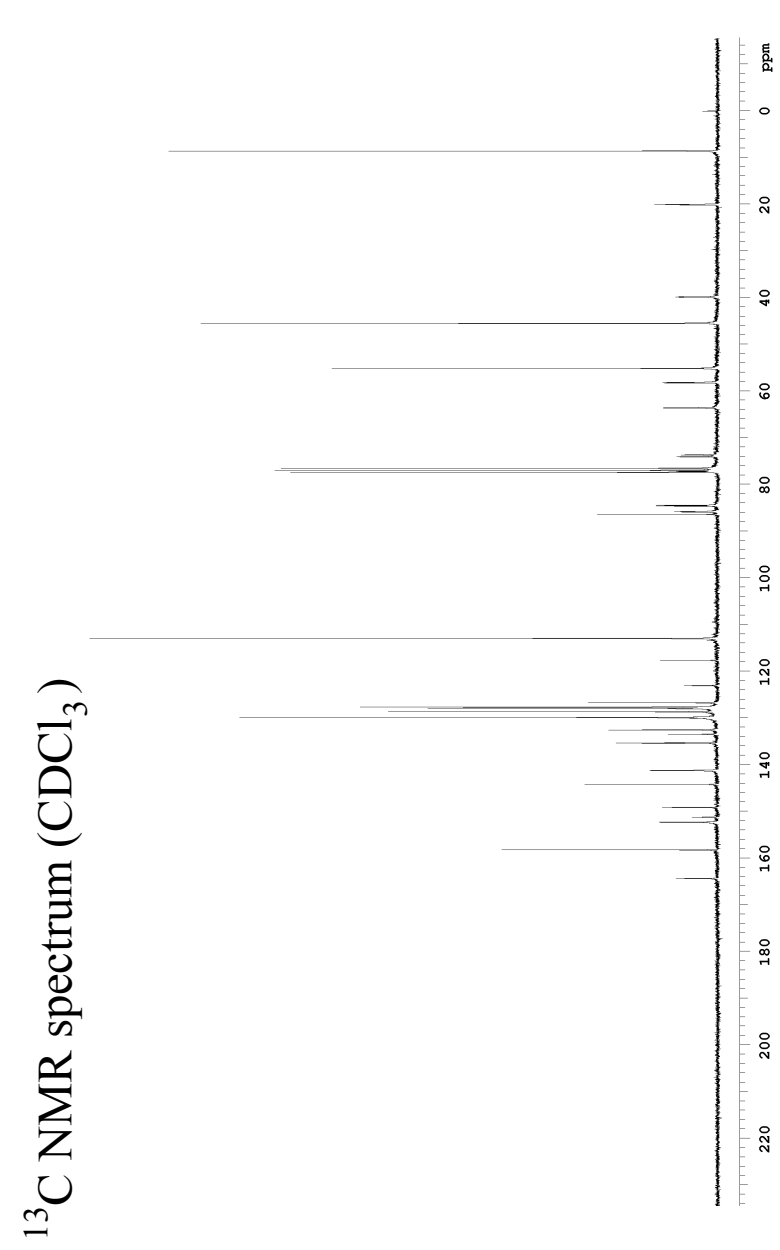

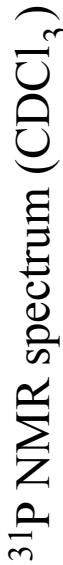

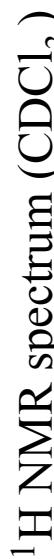




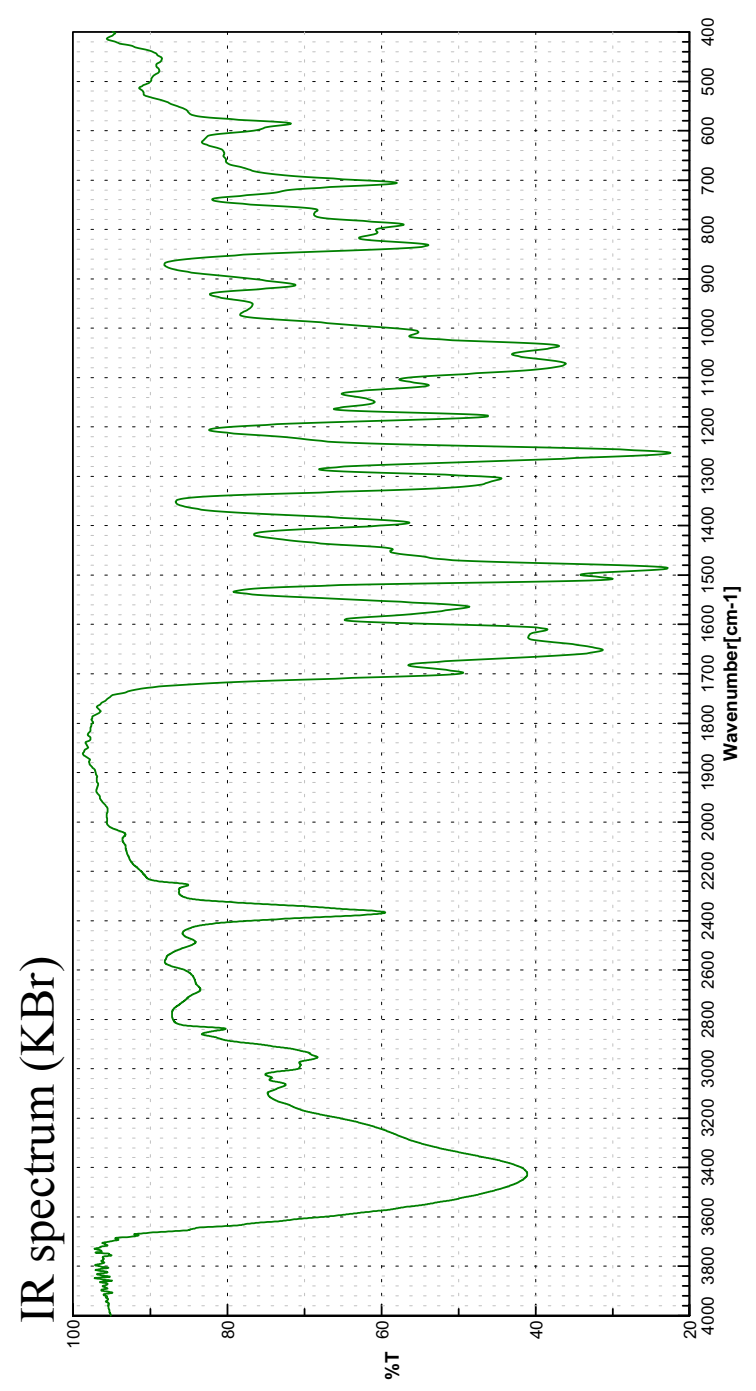

Supporting Info Page 7
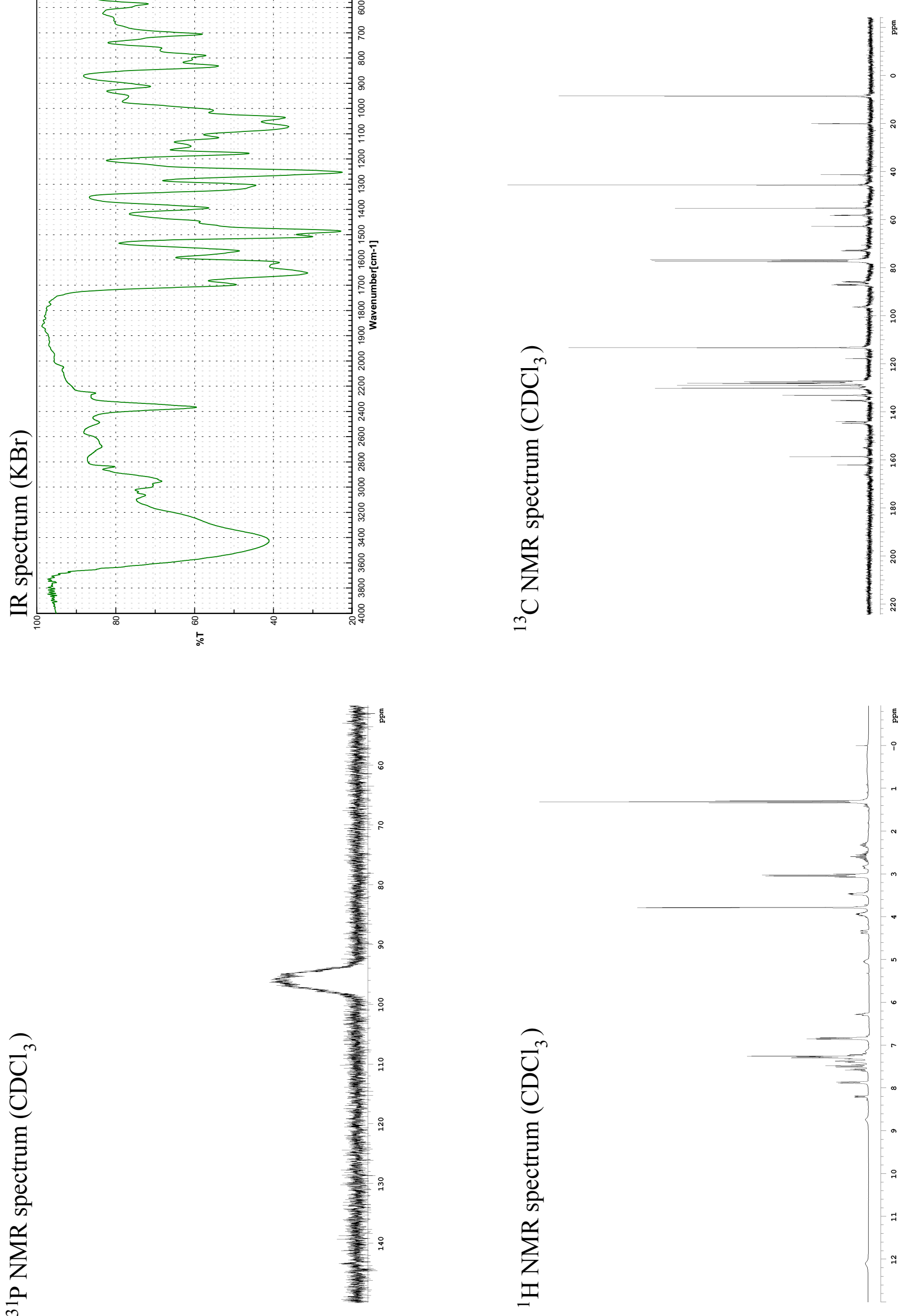

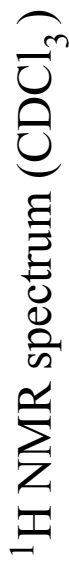




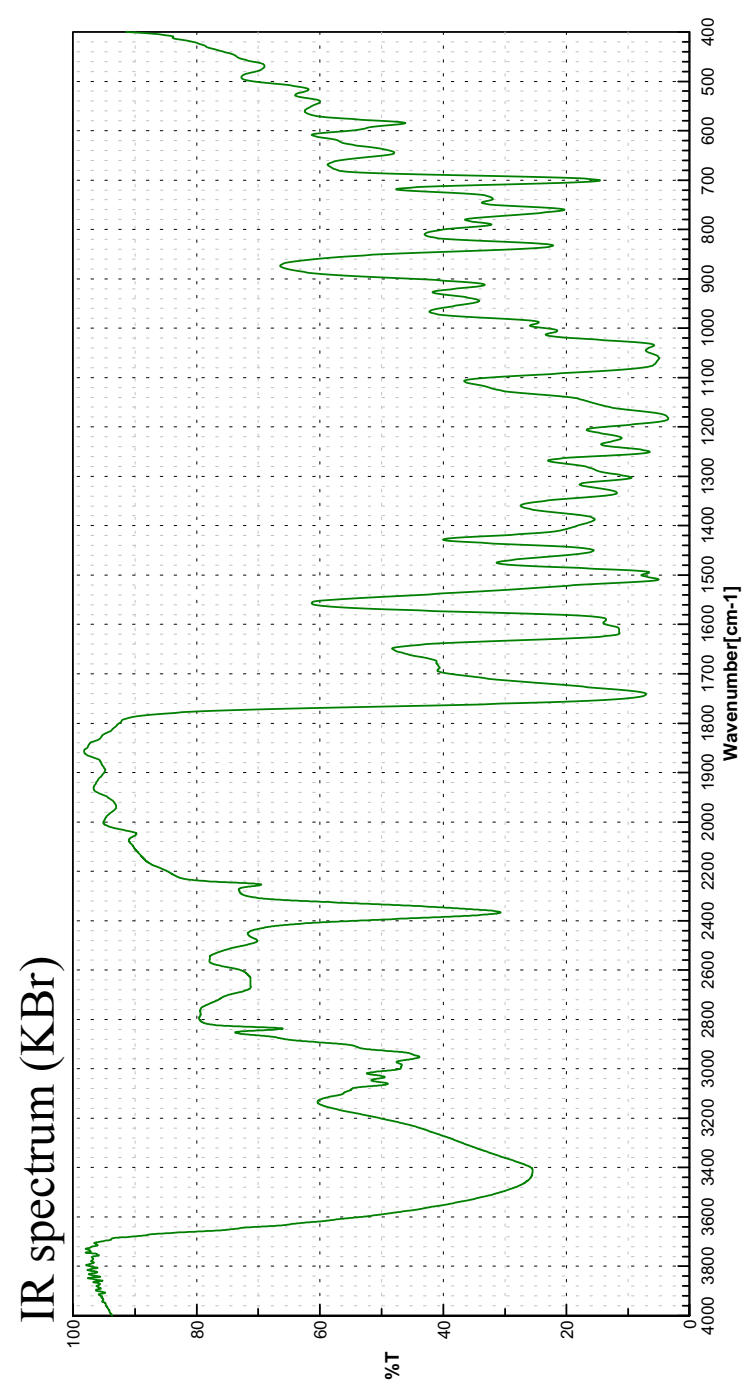

Supporting Info Page 8

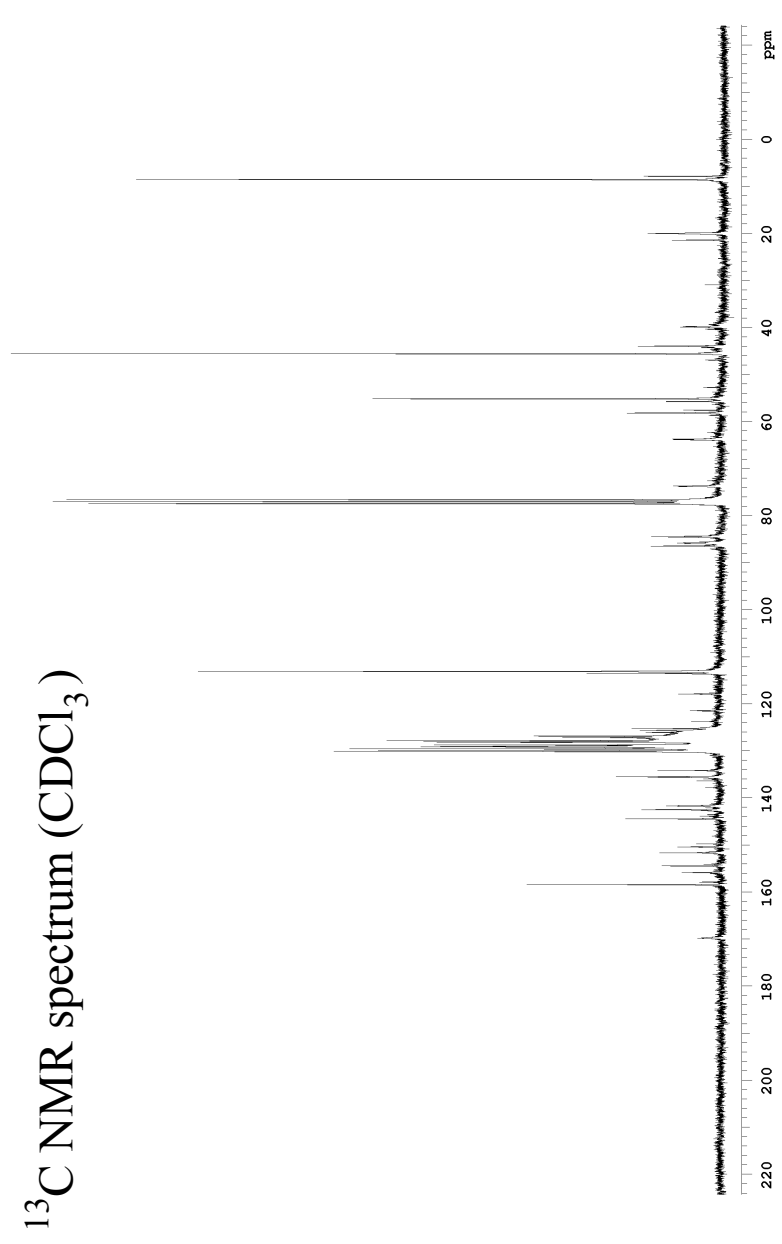

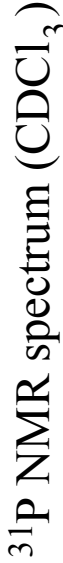

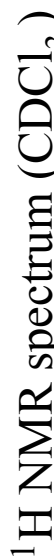




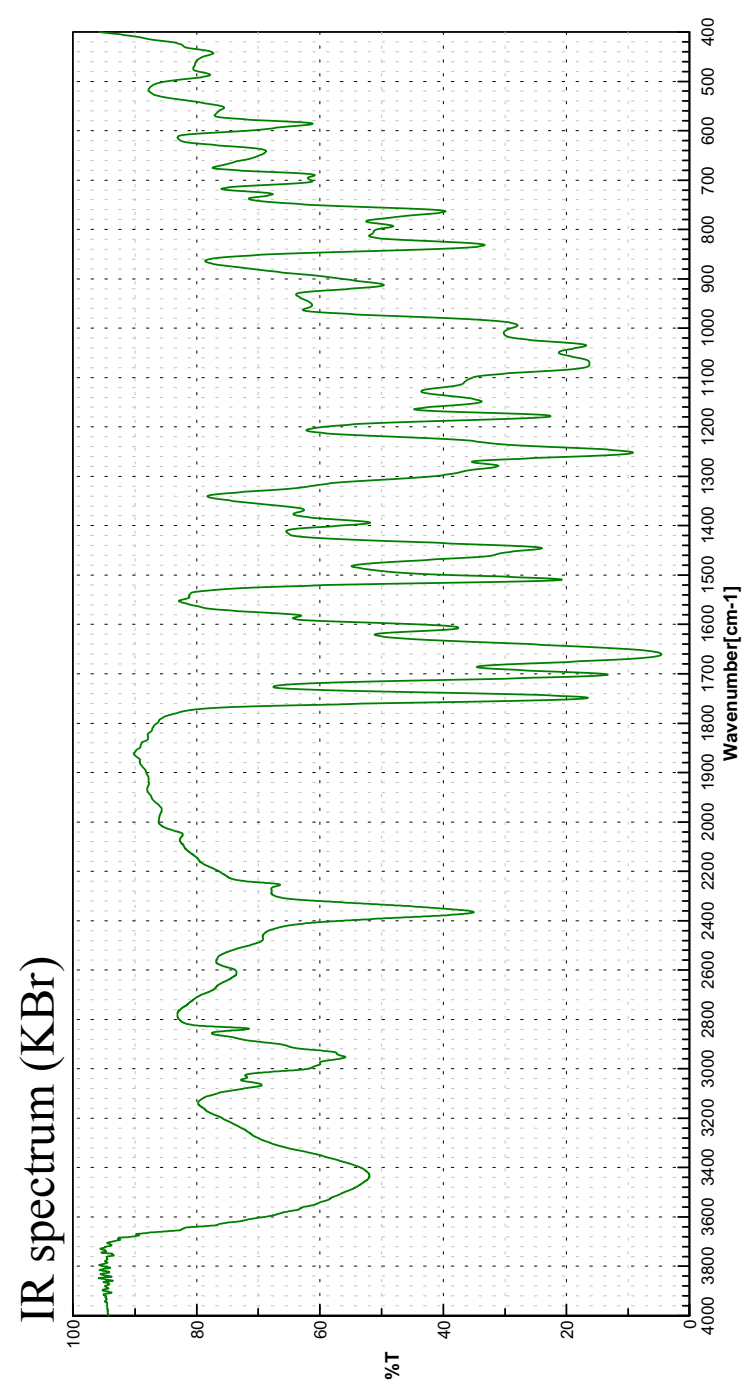

Supporting Info Page 9

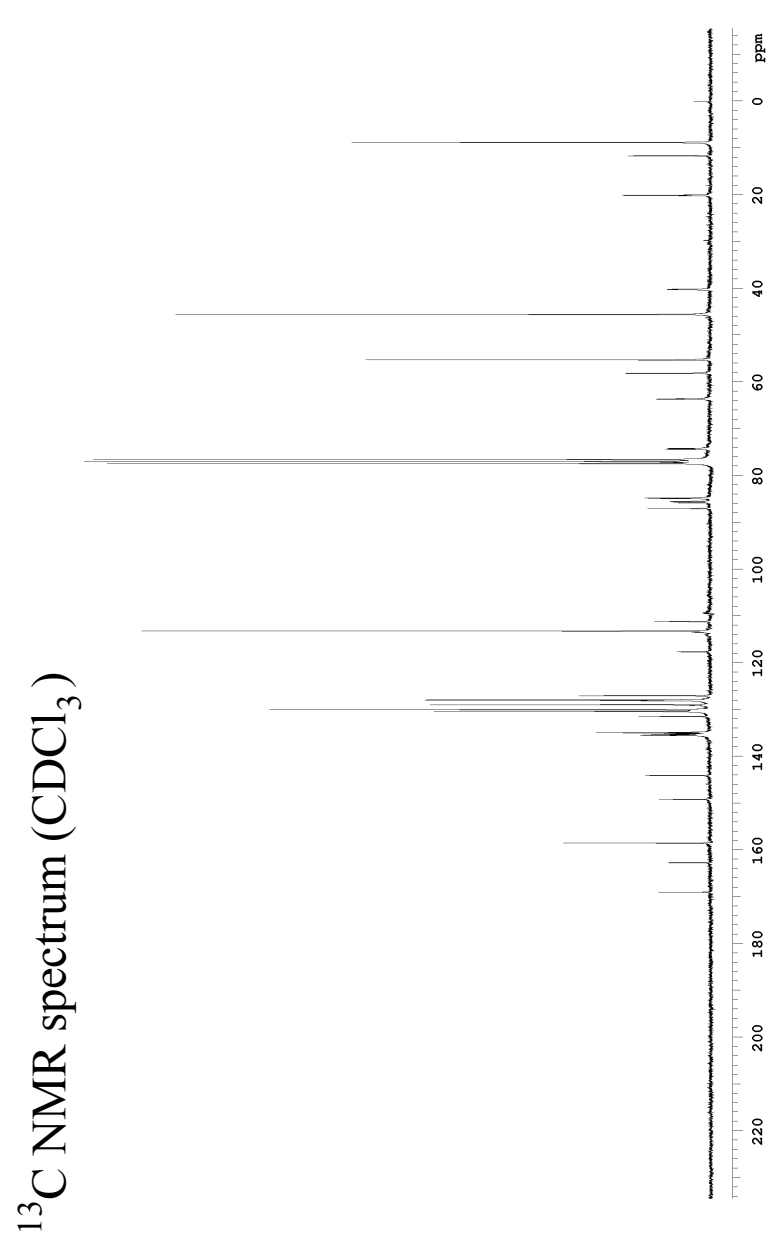

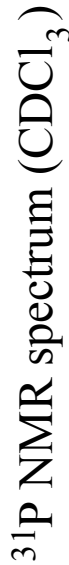

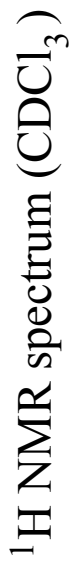




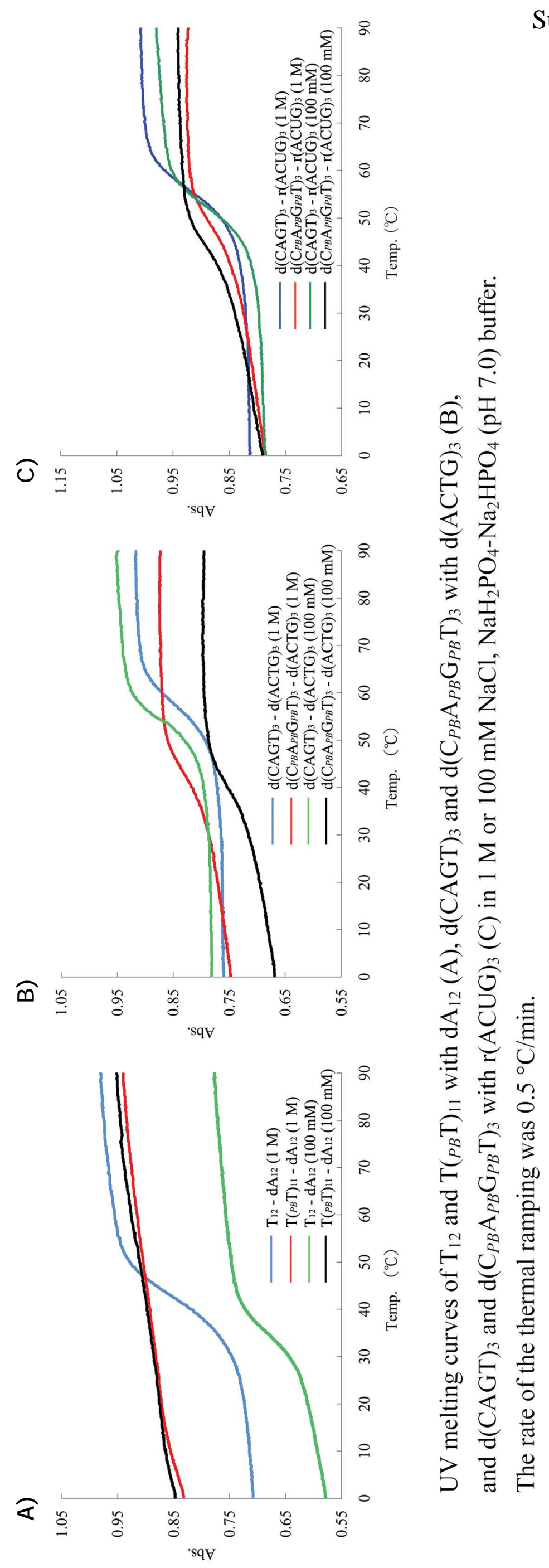

